\title{
How have administrative sources been used to adjust population estimates and set plausibility ranges?
}

Rebecca Tinsley

Office for National Statistics

\section{Abstract}

This article reviews existing methods employed by various countries in the use of administrative data to make adjustments to, or set plausibility ranges around, population estimates or census data. The work was carried out to explore techniques that could be used by the ONS in application with population estimates. An annex also covers benefits and difficulties that have been experienced when producing a register-based census, or population estimates. 


\section{Introduction}

This article explores what evidence exists about approaches to plausibility ranges based on administrative data that have been used or proposed for population estimates, focusing primarily on methods. It will cover, where documented, the strengths and limitations of the methods used. The article will examine international examples of where administrative sources have been used to adjust, improve or quality assure population estimates or census outputs, alongside work done by academics, local authorities, the Office for National Statistics (ONS) and other National Statistics Institutions. An annex covering benefits and difficulties with producing register-based censuses and population estimates has been included.

A paper that presents a selective review of international practice in carrying out census designs that differ from the traditional model used currently in the United Kingdom, including the use of administrative data, is due to be published by the ONS later in the year. Readers may also wish to be aware of an article previously published in this journal which looked at the validation and/or adjustment of Census data across different countries, and included reference to the use of administrative sources ${ }^{1}$.

\section{How are plausibility ranges used?}

To date, no evidence has been found to suggest that other countries are producing plausibility ranges with which to adjust their population estimates. Some of the literature has, however, identified some techniques that have the potential to be used to produce plausibility ranges.

Charlton and Chappell $(1999)^{2}$ described sources of uncertainty in the 2001 demographic estimates (which were based on 1991 Census data). They attempted to estimate possible sources of bias and increased variance due to non-sampling error for each of the components. They used the Delphi Method to do this (Linstone and Turnoff, $1975^{3}$ ). The Delphi Method, described below, was developed for groups to solve effectively a complex problem. It involves repeatedly asking a group of independent experts to provide their best estimate/solution to a problem. The intention is that by repeatedly asking the question and sharing the response of others in the group, a consensus is formed. This is an established method used to help in setting the assumptions for the UK national population projections, produced by ONS.

Charlton and Chappell used this method to obtain a best estimate of bias and the effect of this bias on the error of the estimate for international immigration and emigration, internal migration (to England and Wales and from Scotland, Northern Ireland and the Isle of Man), and to produce an estimate for uncertainty around Asylum Seeker and Visitor Switcher data. To do this, they consulted with a range of topic experts. The experts were asked for their best estimate of bias and error on their topic area of expertise via a questionnaire. The results were compiled and added to a new version of the questionnaire. The recipients were asked to review (and if necessary revise) their estimate, based on the responses from the rest of the group. The intention of this technique is that this process continues until a general convergence of opinion emerges, a respondent group fatigue factor becomes apparent, or a point of diminishing returns is reached.

The Delphi Method could potentially be applied to plausibility ranges: experts could be consulted on their highest and lowest estimates of population (or indeed a component) within a given area, in order to obtain plausibility ranges. This method also has the potential to be applied to 
administrative sources to estimate the bias and uncertainty in the data source and create plausibility ranges around the data source with which population estimates are compared. The advantage of this method is that it draws together expert opinion on the accuracy of the administrative data source or population estimate using a recognised methodology. The disadvantage is that it can be perceived to be subjective, and the credibility of the experts would need to be established.

Some of the research in this article illustrates techniques used to produce population estimates based solely on administrative data. It may be possible to apply some of these techniques to create plausibility ranges with which population estimates could be compared. Evidence from countries that have moved to register-based censuses describes how, in the transition stages, administrative data are used to compare with the developing population register. This approach could help form the basis for plausibility ranges. For example, school census data might be used as a minimum count of school-age children in a given area, if it were deemed a suitable source to use as a minimum.

\section{Approaches being taken for the quality assurance of Census 2011}

ONS has developed a methodology for the quality assurance of Census 2011 population estimates $^{4}$. The quality assurance will be carried out at local authority (LA), regional and England and Wales level, and core checks will set tolerances around a variety of comparator sources which will be used to quality assure the Census estimates. These tolerances will be used to identify areas where more detailed 'supplementary' quality assurance is needed.

Using tolerances recognises that although comparator data provide a good estimate of the indicator in question, the comparators themselves will contain error and variance. There are also definitional and coverage differences. For instance, the School Census does not include children in independent schools.

There are four types of method used to set tolerances:

- Diagnostic range (multiple comparators)

- Quality assessment (single comparator)

- Set percentage (single comparator)

- Change over time (single comparator)

\section{Diagnostic Range}

The diagnostic range approach was first used in the quality assurance of the 2001 Census estimates of age and sex. The same basic approach will again be used in 2011 for a number of checks. The diagnostic range is defined as 'the midpoint of the range of different comparators plus or minus the range itself'. By defining the diagnostic range in this way the range is broadly between 2 and 2.5 standard deviations beyond the extreme values of the range of comparators.

For the 2011 Census quality assurance, diagnostic ranges are calculated for each LA and each category of the variable checked. In the case of age and sex, for instance, different diagnostic ranges are calculated for each five-year age group. Differences in the width of the diagnostic ranges reflect variation in comparator sources between LAs and between age/sex groups. 
There are two refinements made to the diagnostic range approach when applied to age and sex. These are:

1. Comparator data are used as a lower bound rather than the diagnostic range where sources of sufficient quality exist. Patient register data is used as the lower bound for under 1 year olds and the School Census is used for 5 to 15 year olds.

Replacing the lower bound of the diagnostic range with these two sources, the quality assurance process will in effect control these values. Development of the quality assurance approach showed that using the sources in this way narrowed the diagnostic range in almost all cases.

The School Census (for school-age children) and the Patient Register (for babies under one year of age) are both deemed to be of high quality, and sufficiently accurate to use in this way.

2. Where the diagnostic ranges vary greatly, diagnostic ranges are amended to ensure that ranges are not implausibly wide or implausibly narrow.

Amending the ranges involves using cut-off values to remove the very widest and narrowest ranges based on the latest available data, mid-2009. These cut-offs are based on the width of the diagnostic range in an age/sex group for all local authorities, expressed as a percentage of the mid-point. They are identified as the 10th and 90th percentiles.

A similar amendment was made in preparations for the 2001 Census quality assurance. However rather than using broad age groups (0-19, 20-24, 25-29, 30-79, 80-84, and 85+) as in 2001, cut-offs will be calculated and applied in five-year age groups. This refinement is in line with how the diagnostic range will be used, by five year age groups rather than in broad age groups.

\section{Quality Assessment}

Where only a single comparator dataset is available, it is not possible to use the diagnostic range approach. For instance, the number of higher education students will only be checked against data from the Higher Education Statistics Agency (HESA) (combined with data on further education students).

In such cases tolerances have been set after an assessment has been made of the quality and coverage of the comparator data. Quantifying quality and coverage is inevitably difficult in the absence of a comprehensive independent source (such as the Census) against which to compare.

Local authority users were consulted during the process of identifying and quantifying issues of quality and coverage. As part of the quality assurance procedure, summaries of eight of the key administrative data sources were sent to the 30 local authorities involved. The authorities were asked to comment on the issues identified and provide evidence of the size of their impacts.

\section{Set Percentage}

In some cases it proved particularly difficult to set tolerances in relation to the quality and coverage of the comparator. Typically these issues were not only difficult to measure but also varied 
between areas. Where this was the case, it has been necessary to use set percentages above and below the comparator.

Armed forces (home and foreign) checks both use set percentages. Comparator data are available on the number of armed forces personnel stationed in each LA, but not where personnel are resident. Although an adjustment is made to the comparator to indicate residence, this is based on the 2001 Census and so will inevitably be out of date. Tolerances have been arbitrarily set at $+/-5$ per cent around the comparator but will be reviewed during the quality assurance process.

\section{Change Over Time}

The fourth approach sets tolerances in relation to past trends in comparator data. The approach is used for checks where there is a single comparator data source and a time series of data available. To use the change over time approach, there was a requirement for the comparator to be particularly well understood. Mortality and fertility rates are both routinely produced by ONS at LA level, as are estimates of internal migration based on patient registration data.

There was also a requirement for the comparator data to be of high quality. Births and deaths registration data are both of very high quality as registration is compulsory.

\section{Evidence from countries using or considering a register-based census}

Many countries now carry out a register-based census instead of the traditional long-form census carried out in the UK. As part of the transition process, there is evidence detailing how countries have used administrative sources to adjust, or quality assure, their census counts in their progression to creating a population register. Some of these techniques have the potential to be applied to producing plausibility ranges. Benefits and difficulties with producing register-based censuses compiled from papers referred to in this section can be found in Annex A.

\section{Singapore}

Singapore ${ }^{5}$ moved to a register-based census in 2000. Part of the development process involved using annual population estimate data to quality assure the population counts obtained from the register. Where differences were found between the two counts, administrative sources were used to corroborate the plausible estimate, as outlined below. This is one of many examples in this section where administrative sources have been used to identify shortfalls or over-estimates of different population groups.

The check identified some age groups for which the geographic distributions differed between the two outputs; they were consequently adjusted using information from the specified administrative sources:

- 0-4 year olds - many children in this age group had blank address information. A programme was written to link the child to the mother's records and impute the address of the mother. When the children reach school age, their address will be updated with information from school records, which were also used for all children with missing address information. This supports how record-linking and school address information could be used to check geographic distribution of estimates of school age children

- Adults aged 75 and over - the register produced higher numbers of this age group compared to population estimates. To determine which source was more accurate, information from old 
people's homes and institutions for the elderly, in addition to a survey conducted specifically to look at address information of the elderly population showed that many elderly people return to their home countries, or die overseas. The address information was used to update the register and reduce the overcount. This provides another example of how administrative sources are used to verify estimates, but also that survey data could also be incorporated into this process

A range of problems were encountered in the transition between the traditional and the registerbased census, which can be found in Annex A. Of direct relevance to this article are:

- Ensuring that the administrative source covers the same definition that is being counted by the population estimate/register

- Improving cooperation between government departments to improve data sharing and recordlinking - this process also involves applying stringent confidentiality and security measures

- Aligning definitions and classifications across Government

- Identifying and correcting erroneous records and coverage

- Ensuring that all sources used relate to the same time-point

\section{Italy}

Italy $^{6}$, which is moving towards its first register-based census in 2011, also encountered similar problems in developing a register. Pilot work was carried out in 2009 to compare the integrated population register with demographic population estimates for each municipality. Where large discrepancies were identified, they were subject to further investigation, although detail about those investigations is not outlined. It is unclear from the source how 'large' was defined: the absolute difference was calculated between the two sources.

Lessons learned from this process were that discrepancies existed between demographic estimates and administrative data for the following reasons:

- Differences in reporting period for the two sources (this was problematic as people may move house between the dates and deaths/births could occur in the interim period)

- Missing data

- Differences in classification/coding of variables between sources, or between the same source if collated separately at the local level

- Duplication of records for an individual

Italy is using administrative data to improve the register's capture of migration at a local level, as it has identified both overcounts and undercounts of migrants. This is thought to be due to there being: 1) no legal requirement for migrants to register a change in address, 2) no formal registration for leaving the country, and 3 ) delays for migrants to register when they arrive. An experiment was carried out to assess whether 'permit of stay' data could be linked to the register, and whether this data could improve the quality of migrant data ${ }^{7}$.

Despite this data source under-representing children aged under 18 (as children are often under the charge of an adult and therefore have no requirement for a permit), it was found that the data was able to improve both errors. Undercount was improved by those migrants who appeared on the permit to stay data, but not on the register (due to not yet having registered, but who would 
become usual residents), and overcount was rectified by using the date of expiry on the permit to identify those who had left the country.

Whilst this method is not applicable in the UK as the latter has no register with which to link, and has no similar source (until the advent of e-borders), ONS could consider the possibility of combining administrative sources to produce upper and lower thresholds as plausibility ranges.

\section{Israel}

Examples from Israel ${ }^{8,9}$ show different sources of administrative data that could be considered, in addition to supporting further the argument that combining data sources can improve estimates of population based on administrative data. Israel produced its first integrated census in 2008. This involved using a combination of administrative data sources which were merged into a comprehensive file called the 'Improved Administrative File' (IAF), and a detailed survey on approximately 20 per cent of the population. The survey provides more detailed information, such as socio-economic status, which cannot be captured by the register.

The key source used in the IAF was the Population Register (PR) that already exists in Israel, and which holds personal, demographic and address information on each citizen, as well as information about relations between family members. In order to validate, and improve the quality of address information in the PR, it was merged with three independent administrative sources:

- Pupils on the student register (covering elementary, secondary and high schools)

- Electricity company accounts

- Driving licences

It was possible to link this accurately using a person's unique Registry Identification Number (RIN). Presence in the country was corrected using information from border control files.

\section{United States}

The next two sources show how the United States is using administrative data to estimate population. The first outlines an experiment linking administrative data to an address register to assess the feasibility of supplementing or replacing the census. This provides evidence of which data sources are being used, and also highlights a possible technique that ONS could consider to set plausibility ranges. The second outlines how an address register could be expanded to contain population information, thus replacing population estimates. This is done through linking to administrative sources, survey data, and simulation techniques.

Following various debates in the United States about how to overcome non-response for the 2000 Census, alternative options were proposed including using administrative data as a direct substitution for non-response households, and simulating a complete 'administrative records census'. Judson (2002) ${ }^{10,11}$ outlines the Administrative Records Experiment (AREX 2000) which was carried out in 2000 alongside the Census as a feasibility study for whether administrative data could supplement, or replace, the Census. AREX was carried out in Maryland and Colorado. Two approaches were considered: top-down and bottom-up; a combination of these approaches was used, as outlined below. 
A range of administrative sources were used in AREX 2000, including Internal Revenue Service (IRS) tax data, Tenant Rental Assistance Certificate System data, Medicare enrolment data, Indian Health Services patient registration data and Selective Service System registrations.

The AREX starts with the Master Address File (MAF) or Census 2000 Address List. The latter is a comprehensive list of addresses in the US, produced through a combination of US Postal Service data, 1990 Census address list, Census 2000 field operations and local address lists and files. Administrative records are then linked with the MAF, leading to three linkage outcomes:

1. administrative records linked to a MAF address

2. administrative records not linked to a MAF address

3. MAF address unlinked to administrative records

Top-down enumeration was carried out for those administrative records not linked to a MAF address through cleaning and validation, while bottom-up enumeration was carried out to add persons missed in the administrative records. It could be possible to consider applying this technique to the production of plausibility ranges. For example, record-level data from one data source could potentially be used as one bound, and aggregate-level data from another source could provide a range of plausible estimates for a given population.

The AREX was found to have some limitations, such as access to only a limited range of administrative sources, the experiment being carried out on a small number of test sites, and poor quality recording of race and Hispanic origin. Despite this, plans were made for an improved AREX in 2010, to include:

- Greater coverage

- More timely data sources

- Additional sources (including benefits data)

More recently, Swanson and McKibben (2010) ${ }^{12}$ outlined a proposal for producing US population estimates using an enhanced version of the Master Address File (EMAF), detailing how this could be used to develop timely, cost effective and precise population estimates for very small geographic units. They propose that as American traditions and values do not support a population register, EMAF could be an alternative option.

The ultimate plan for this approach is to produce occupancy status, demographic and socioeconomic data through a combination of decennial census, American Communities Survey and administrative data sources, through record-matching, imputation and micro-simulation methods. This data would then be updated each year, and every ten years it would be recalibrated to the decennial census.

Swanson and McKibben identified three main obstacles to this approach, two of which are more relevant to producing an administrative source-based census and are therefore outlined in Annex A. The third relates to accuracy and technical challenges which need to be taken into account when using administrative data for population estimates, such as:

- Resolving address-related issues (duplicates, omission, deletion and incorrect locations) 
- Implementing GPS-based geographic coding of housing units

- Problems relating to how seasonal populations and seasonal housing make it harder to produce an estimate of a de jure population

- Problems associated with rural addresses

- Issues with counting populations that don't have a standard address (such as institutionalised, homeless and transient populations)

- Systematic biases in administrative records

- The need for a set of unified id codes to match, and merge records from different administrative systems

Many of the benefits for this method are listed in Annex A; these relate more to producing population estimates based entirely on administrative sources. However the following are pertinent to the theme of this article:

- Improved estimation of both internal and international migration

- An improved source of data for users

The remaining sections outline UK and non-UK research surrounding the use of administrative data to produce a population estimate, or component of population, that may have a practical application to adjusting or setting plausibility ranges for UK population estimates.

\section{Evidence from using administrative sources to estimate population}

\section{United Kingdom}

The following outlines how a consultancy company produced population estimates for a range of local authorities, using administrative sources. This shows which combinations of administrative sources have the potential to be used to produce estimates, and which sources have the potential to be used for 'reasonability checks' when quality assuring the data.

Following the results of the 2001 Census, some LAs suggested that the results were subject to under-count ${ }^{13}$. Some authorities such as Newham, Hackney and Ealing used locally-held data sources such as GP registers, school registers and NINo (National Insurance number) data to estimate their populations. Among others, Brent, Hackney and Newham engaged an independent consultant to carry out population estimates based on administrative sources. The company was asked to adapt their data linking 'neighbourhood knowledge management' (NKM) technique in order to produce alternative population counts for Hackney ${ }^{14}$.

A cleaned version of the GP register provides the basis for the population estimate. The clean register provides a base 'minimum confirmed population' with each record representing a confirmed current resident, their age, sex and address. This data is then matched to an address list, in this case the Hackney Local Land and Property Gazetteer (LLPG) was used. The LLPG contains a Unique Property Reference Number (UPRN) for each address on the register; this number is then assigned to each address on each of the administrative sources that are to be used. In this case birth and death records, the electoral roll, council tax liable persons and benefit recipients, school pupil registers and housing tenure data were the administrative sources used. 
The assignment of the UPRN enables ease of matching between the sources. The administrative sources were then used to determine whether a person is a current resident at a given UPRN.

The next step involves person-matching to check the same person is identified across each data source. Due to the lack of a unique person identifier, a fuzzy-matching process is applied. The final stage assimilates the information learned about each person (such as age, gender and address) into a core database from which population information can be extracted.

The quality of the population estimates produced from this methodology was assessed through a selection of 'reasonability' checks with data from other sources; the checks carried out were:

- The number of children aged 0-16, which was compared to DWP data on Child Benefits

- Single occupancy rates which were compared to those produced in 2001 Census

- The vacant address rate which was compared with the LA's own data including Council Tax records

- Addresses with high occupancy levels (in this case, properties with greater than nine people) were checked with genuine multiple occupancy properties such as hostels, retirement homes or student halls of residence

- A check on the number of children aged under 16 who lived at an address without an adult

These checks were all found to produce estimates considered to be within acceptable margins of error (although there is no explanation of how 'acceptable margins of error' are set). In addition, the population estimates were compared to both the Greater London Authority (GLA) projections and ONS Mid Year Estimates (MYEs). The administrative estimates produced population totals higher than both the MYEs and the low GLA projections, showed similar age-sex distributions, and also produced significantly higher estimates for the 20-24 age group.

Advantages of this methodology are that data can be produced for any geographic level as all addresses are geo-referenced (have $x-y$ coordinates) and the final database is a very rich source of data which can be linked to many different data sources and used to produce data for a variety of different population problems (recent case studies have included teenage pregnancies, regeneration and well-being). A paper by Verrall $(2009)^{15}$ outlined research suggesting that the methodology 'is more cost-effective, up-to-date and fit for local analysis than traditional population statistics'.

Rees et al $(2004)^{16}$ discussed the use of adjusted electoral register data to count the population of small areas. Currently, Electoral Registers (ERs) have an indicator for those aged over 70 (as they are no longer eligible for jury service), but contain no other age information. Other groups are also not contained within ERs, such as those aged under 18 and foreign nationals who are not eligible to vote at the next election, those who fail to register, those who are bankrupt, prisoners and those who are clinically insane. ERs therefore need adjusting to take account of these populations if they are to be used to produce estimates of population.

In order to estimate the number of those ineligible to vote due to their age, a ratio was calculated as the proportion of those aged under 18 compared to those aged 18 and over, based on 2001 Census data for each ward. This ratio was then applied to the electoral count for those aged 18 and over in order to estimate the number of those too young to vote and therefore not on the 
register. Whilst it may be more appropriate to look at school census data for a plausible estimate of the number of children in the area, this technique has the potential to provide an alternative count. It would also take into account those aged 0-4 who would not be covered by School Census data. Additionally, electoral roll data has the potential to be used as a source of information for those aged over 70 .

\section{United States}

There is much evidence from the US about producing estimates at the local level (Smith and Mandell $(1984)^{17}$, Smith $(1986)^{18}$, Murdock and Nazrul Hoque $(1995)^{19}$, Hunsinger $(2010)^{20}$ and Plyer et al $\left.(2010)^{21}\right)$. These papers outline the main techniques that are currently used by different states to estimate small-area populations. Some are used in the UK (ratio change methods and cohort component methods). The other three key methods referred to are the Housing Unit method, the ratio-correlation method and the administrative records method. These methods have the potential to be adopted to produce plausibility ranges.

The Housing Unit (HU) method is calculated by multiplying the number of occupied housing units by the average number of persons per household, and then adding an estimate for the Group Quarters (GQ) population (GQ in the US refers to university dormitories, military barracks, nursing homes, correctional and juvenile facilities; GQ estimates are often drawn from the most recent census or from information provided by administrators of the facilities). The methodology and its limitations are outlined below. This technique could be considered as an alternative way of producing plausibility ranges for population estimates at the local level.

The number of occupied households is usually calculated from administrative sources; usually building permits or certificates of occupancy data, or utility data (such as electricity company or telephone company records). Alternatively, voter registration lists and post office address lists can also be used. Some of the problems experienced with the use of these sources, and which should be considered when selecting sources, are:

- Variation of data availability, dependent on the local area

- Variability of data quality (dependent on the service provider)

- The need to incorporate information from multiple service providers (for example, more than one electricity company)

- The identification of an occupied (vs. non-occupied) residence (especially identifying seasonally occupied residences)

- Reduction in the number of people (especially young people) who have a landline

The average number of persons per household $(\mathrm{PPH})$ is calculated using data from the most recent census. However this assumes little change in household composition, which becomes less appropriate as time passes. An alternative way to calculate this is to use data from more up-to-date household surveys (in the US, the annual Current Population Survey is used). This figure can be used to proportionately adjust the census estimate for PPH (for example, if the local area's census $\mathrm{PPH}$ value was higher than the national/regional census PPH value, the national/regional PPH value from the survey would need to be adjusted with the ratio obtained from census year). A lower bound of 1.0 for the PPH is always applied. 
Alternative methods involve calculating a PPH for each type of household (for example, singlefamily, multi-family and mobile-home units); this can be of particular use to local areas with an unusual mix of housing units (for example exceptionally high numbers of multi-family units). Regression analysis to relate changes to PPH with changes in variables such as births, school enrolments and exemptions per income tax return has also been done to model PPH.

Smith (1986) $)^{18}$ found evidence to suggest that the PPH element can be estimated more accurately than the occupied housing element; possible explanations for this are those problems listed above, and the fact that PPH may remain more stable over time than the number of occupied houses. This is also the only method which produces an estimate for the population aged over 65 without the use of Medicare data.

The ratio-correlation model is a method that uses regression modelling to make estimates of small area population estimates, based on administrative data that are symptomatic of population change. This method is currently being used in Colorado to make a local estimate of population as an alternative to the annual population estimates, in order to be less reliant on the US Census Bureau's administrative records-based migration estimates (Hunsinger, 2010) ${ }^{20}$. This method has the potential to be used to make an alternative population estimate at the local level in the UK.

Ratio-correlation estimates the ratio of population change from state- to county-level from one time period to another, based on regression using state- to county-level ratios of different administrative sources. This ratio is then applied to the base population count in order to produce a population estimate. Administrative sources that are considered to be symptomatic of population, and commonly used in this area are counts from birth certificates, death certificates, school enrolment data, voter registrations, driving licences, occupied housing units, employment data and tax records. Multiple models and dummy variables can be added to improve the modelling (especially if there are area differences which may imply a different relationship between the symptomatic variable and the population count). This method is applied to the non-GQ element of population (it is usually assumed that accurate data for these groups can be accessed on an annual basis).

In the research done by Colorado, counts from birth certificates, school enrolments, vehicle registrations and voter registrations were found to produce a good model fit.

Problems identified with this method are:

- The assumption that patterns of change over time between the symptomatic variables and the Census will remain the same

- The risk of multicollinearity (that is, the risk that two or more of the symptomatic variables may be highly correlated, leading to errors in the interpretation of the model)

An analysis of studies where an estimate of error between estimates produced using ratiocorrelation and census counts showed that ratio-correlation estimates have on average a Mean Absolute Percent Error (MAPE) of around 5 (Smith and Mandell, $1984^{17}$, Murdock and Nazrul Hoque, $1995^{19}$, Hunsinger, $\left.2010^{20}\right)$. This information may be useful for setting tolerances for plausible estimates.

The administrative records method is similar to the component method, except that the net-internal migration element is based on changes in addresses on federal income tax returns. Estimates of 
international migration are made using records from the Immigration and Naturalization Service, then changes of address in Medicare enrolment (for the over 65 population) and GQ addresses are added in to provide total population estimates.

Comparison of these different methods has shown some evidence of undercount, but found Housing Unit and ratio-correlation methods to produce the lowest levels of undercount and smaller errors. It may also be noteworthy that US official estimates of population at both state and county levels use an average of estimates produced using ratio-correlation, component change and administrative records methods.

\section{Evidence from using administrative sources to estimate components of population estimates}

\section{United Kingdom}

Boden and Rees (2009) ${ }^{22}$ proposed two models for distributing Total International Migration (TIM) estimates to Government Office Region (GOR) and LA level. This technique could potentially be used to produce a plausible range of migration data at LA level.

The first approach involved using GP registration data to distribute the TIM estimates, while the second proposed using three different administrative sources. This approach proposed the use of NINo, HESA and GP registration data. The allocation of the TIM estimates to these sources was based on the 'reason for migration' data published in the 2007 ONS International Migration series. This found that 26 per cent migrated for study (and are therefore assumed to be students and can be distributed via HESA data), 40 per cent migrated for work (and can therefore be distributed by NINo data), and 34 per cent stated reasons for migration as accompanying or joining family members, 'other' or did not state their reason (for this group, GP registration data will be used to distribute them). Boden and Rees agreed that the second was the preferred approach as it reflects more complex migration patterns.

Key findings of this analysis were that, at GOR level, there was increased allocation to the West Midlands and London (and to some extent, the two GORs in the North), while there was a decreased allocation to the East of England, Yorkshire and Humber, and the South West. This analysis suggests that some local consultation would be needed to refine the model, especially for those areas where the LA distributions differ significantly from those of ONS. For example, LAs showing large changes were often cities with universities which would need to be taken into account.

ONS has recently developed a new methodology to distribute immigrants to local authorities using a range of administrative data. This new approach builds on some of the thinking of Boden and Rees and is now feasible because of recent improvements in ONS access to, and understanding of, administrative sources such as the Higher Education Statistics Agency (HESA) data for students, the Migrant Workers Scan (MWS), and the Lifetime Labour Market data base (L2) for workers. These administrative sources (apart from the L2) are not based on a sample and so can be used to produce estimates for small areas. However, there are other issues such as differences in coverage and definitions as well as overlaps between different sources. The development of the improved methodology takes account of these issues. 
Smith, Raymer and Guilietti $(2009)^{23}$ wrote a paper outlining three sources that could be used to model internal migration by economic activity at county level. The three sources were the Patient Register Data System (PRDS), 2001 Census and Labour Force Survey (LFS) data (the latter is an extension to the framework proposed by Raymer et al $\left(2007^{24}\right.$ and $\left.2009^{25}\right)$, which used PRDS and Census, but not LFS data, and which was at a higher geographic level). A log-linear approach was taken to model data to origin, destination, age, sex, economic activity and time period. The outcome of this research shows that migration patterns vary for different economic activity groups. This shows that there may be a need to use different sources to distribute migration data in order to take account of these varied patterns; again, this method could be considered to produce a plausible range of internal migration estimates at the local level.

\section{New Zealand}

Statistics New Zealand ${ }^{26}$ use information about significant local developments such as newly built prisons, student hostels, apartment complexes and retirement homes to improve internal migration estimates. The age and sex ratios are adjusted accordingly. Prior to 2004, internal migration estimates were based on population change using two administrative sources: residential building permits and employment data.

Since 2004, the following sources were evaluated and combined to estimate sub-national internal migration:

- Residential building consents data

- Electoral enrolment data (for those aged 18 and over, including short-term migrants); this data is available broken down by age

- Inland Revenue Department data (covering all employed, self-employed and benefit claimants); record-level data available including DOB, address and address for the previous year

- School enrolment data for all schools by age, sex and area

Bycroft (2006) ${ }^{27}$ refers to the use of migration data events (travelling into and out of New Zealand) which can now be matched via passport number. This means an accurate estimation of short-term migrants as the time between entry and exit (or exit and entry) can be assessed. This may be of interest in relation to the future use of e-borders data.

\section{Other examples of administrative data used in the production of population estimates}

\section{Canada}

Statistics Canada ${ }^{28,29}$ estimate the number of emigrants and returning emigrants using Child Tax Benefit data. Internal migration is estimated using Child Tax Benefit data and the annual tax files received from the Canada Revenue Agency.

Canada carries out a census every five years; 80 per cent of households are sampled with a short form questionnaire and the remaining 20 per cent are sampled with a long form questionnaire. Administrative data on the recent growth of housing (for example new sectors being developed) are used to improve the coverage sampling frame. 


\section{United States}

Plyer et al (2009) ${ }^{21}$ evaluated a range of administrative sources that were potentially of use for estimating population following a US catastrophic disaster. The evaluation of suitability of each source was based on the following criteria:

- Soundness (holds up to assumption, that is, constant relationship to population in a postdisaster situation). 'Soundness' was judged on how appropriate data were for measuring postdisaster population

- Consistency (collected in a stable and consistent manner over time)

- Relevance (frequently updated to the appropriate level of granularity required)

- Timeliness (data are available with a minimum time lag)

- Accessibility (easy to access the data, with minimal barriers)

Data sources assessed for Housing Unit method:

- Utility records - electricity records are deemed the utility source with the best coverage in the US (that is, compared to telephone, water or gas). However one drawback is that this is usually a single point for multiple address complexes like flat/apartment blocks

- US Postal Service (USPS) counts of residences actively receiving mail - this may be a sound source except for rural areas or areas with large proportions of seasonal housing. As above, there is usually a single point for multiple address complexes

- USPS national change of address data - as this is voluntary, it is not considered to be as sound as the two sources above

Data sources assessed for Censal Ratio method:

- Driving licences and registered passenger cars - not robust across states, or between groups of people

- Traffic volume - there is no literature using traffic volumes as a basis for population estimates. Traffic volume is also impacted by many other demographic changes such as commuting patterns, shopping or other errand-related trips etc, rather than pure population changes

- Voter registration data - consistency and soundness vary across states

- Voter participation data - not considered to be a sound data source as voter behaviour is very complex, and the reason for voter turnout (or not) are numerous

- School enrolment - this forms a sound basis for the ratio based method, although public school enrolment levels may change over time (that is, more parents may chose private schools), which must be considered

Data specific to damage to housing:

- Housing damage estimates - these are based on remote sensing, and house-to-house inspections. Data can be used to identify areas that remain intact following a disaster, although data availability may vary across areas due to rapidly evolving mapping and remote sensing technologies 


\section{Other examples of administrative data used to produce population registers}

\section{United States}

Sailer et al (1993) ${ }^{30}$ reported on the proposal for using Inland Revenue Service (IRS) data to count the population. IRS data covers individual tax returns and includes any exemptions, children or other individuals that a person is supporting. It also includes information on pensions. IRS data was found to overcount the $65+$ population, even after accounting for deaths occurring over the same period. It was assumed the overcount to be due to some accounts remaining active (and therefore generating information documents) even after the individual died. It was necessary to use vital statistics data on births and deaths to adjust IRS data for those aged 0 , and the $65+$ age group. Concerns were also held about IRS data overcounting due to fraudulent claimants.

IRS data accounted for between 95.2 and 98.4 per cent of Census counts. Age distribution showed an undercoverage of under 30 year olds (especially young women) and an overcoverage of over 75 year olds (especially older men), even after adjusting for deaths. The main undercount was for young children and young mothers on welfare. The older man overcount was thought to be due to the fact that widowed women are legitimately claiming from their husbands' estates, so there is some double-counting (once as the woman, once as the deceased spouse).

\section{Netherlands}

Everaers and Van Der Laan (2003) ${ }^{31}$ wrote a paper on the Dutch system of social statistics, using the process of micro-integration. This system involves linking data from different administrative sources and survey data at the micro, or individual record, level. The benefits of this approach are improved coherence and detail of statistical outputs, increased efficiency of the statistical process, and producing outputs for the best value for money.

Statistics Netherlands developed the Social Statistics Database (SSD), based on the 'life cycle' model of a person. The purpose of SSD is to cover all aspects of a person's life from birth to death, and can be used to analyse the socio-demographic, socio-economic and socio-cultural situation of population groups, and the transitions between social situations. SSD is primarily based on register information, and supplemented with data from business and household surveys which cannot be obtained from register data.

Additional benefits of the micro-integration system are that it can also be used to quality assure the coverage of register data; imputation has been used where a small number of cases was identified as missing. The system also acts as an effective way to counteract non-response bias as it is possible to search for the characteristics that correlate highly with the probability of the response, and with the target variables in the survey.

Poland ${ }^{32}$ will be adopting a similar approach in their 2011 Census, where it is planned that approximately 28 sources of administrative and survey data will be integrated at the micro-level.

\section{France}

In France ${ }^{33}$, the rolling census sampling frame is the Repertoire d'Immeubles Localises (RIL) which is a residential buildings inventory based on 1999 Census data. This inventory is regularly updated using administrative sources such as building permits, local tax planning data and Post 
Office address files. All local communities, such as retirement homes, residential schools, prisons, religious community groups etc, are comprehensively enumerated via the Communities Inventory. This is also based on 1999 Census data and is updated using administrative sources such as registries on healthcare institutions, residential schools and prisons. Each year, the RIL and the communities register are assessed by each local area, where erroneous records can be identified and updated.

\section{Conclusion}

The main conclusions that can be drawn from this synthesis is that the UK would be a front-runner in the production of plausibility ranges, in order to make comparisons with population estimates. However it is becoming common practice to use administrative data in the production of both population estimates and census data. This can be seen through both the number of countries who have chosen to move towards a register-based census (or those who are assessing the feasibility to do so), and the number of local areas who are using administrative sources to make their own assessments of population.

This article has identified a range of techniques that could potentially be applied to the production of plausibility ranges:

- The Delphi Method

- The use of single, or combined administrative sources to set plausibility ranges

- The use of survey data (in combination with the above)

- Using a combination of record-level and aggregate-level data to set ranges

- Using different techniques to produce an estimate of population, which could then be used to make some assessment of plausibility (examples are using new sources to distribute data, the Housing Unit method, ratio-correlation method, neighbourhood knowledge management technique and enhanced versions of the electoral roll)

The article also draws together limitations found when using administrative sources, which must be considered prior to being used. These are:

- Variability in the quality of sources

- Variability in coding/classification of variables between sources

- Variability in the quality of a data source from a spatial perspective

- Issues relating to identifying a household that is occupied vs. a seasonally-occupied, or unoccupied residence

- Ensuring data relate to the same time period

- Resolving address-related issues (duplicates, omission, deletion and incorrect locations)

- Issues with counting populations that do not have a standard address (such as institutionalised, homeless and transient populations)

- The need for a set of unified identification codes to match and merge records from different administrative systems 


\section{References}

1 Newell, R. and Smallwood, S. (2010). A cross country review of the validation and/or adjustment of census data. Population Trends 141: 115-129.

2 Charlton, J. and Chappell, R. (1999). Uncertainty intervals for the 2001 National Demographic Estimates. ONS(ONC(SC))99/05.

3 Linstone, H. A., Turnoff, M. (eds). (1975). The Delphi method: techniques and applications. Readind MA, Addison-Wesley. http://is.njit.edu/pubs/delphibook/delphibook.pdf

4 Office for National Statistics, UK. (2011). 2011 Census- Methodology for quality assuring the Census population estimates. www.ons.gov.uk/ons/guide-method/census/2011/the-2011census/processing-the-information/data-quality-assurance/2011-census---methodology-forquality-assuring-the-census-population-estimates.pdf

5 Statistics Singapore (2001). Combining survey and administrative data for Singapore's census of population 2000. 53rd session of the International Statistical Institute, Seoul, 2001. Online at: www.singstat.gov.sg/pubn/papers/people/cp-census082001.pdf

6 National Institute of Statistics, Italy. (2010). Using population registers as a population list: some results from the Pilot Survey 2009. Joint UNECE/Eurostat Expert Group Meeting on Register Based Census.

$7 \quad$ National Institute of Statistics, Italy. (2010). The use of permits to stay to check the local population registers undercount. Joint UNECE/Eurostat Expert Group Meeting on Register Based Census.

8 Central Bureau if Statistics, Israel. (2010). Evaluating the integrated census in Israel. Joint UNECE/Eurostat Meeting on Population and Housing Censuses.

9 Kamen, C. S. (2005). The 2008 Israel Integrated Census of Population and Housing. Central Bureau of Statistics, State of Israel.

10 Judson, D. (2002). Merging administrative records databases in the absence of a register: data quality concerns and outcomes of an experiment in administrative records use. U.S. Census Bureau. Conference of European Statisticians, Joint UNECE-Eurostat work session on registers and administrative records for social and demographic statistics.

11 Judson, D. H. and Bauder, M. (2002). Evaluation the ability of administrative records databases to replicate Census 2000 results at the household level. Joint Statistical Meetings, Section on Government Statistics.

12 Swanson, D. A. and McKibben, J. N. (2010). New directions in the development of population estimates in the United States. Population Research and Policy Review, 29, 797-818. 
13 House of Commons, London Regional Committee. (2010). London's population and the 2011 Census. Online at: www.publications.parliament.uk/pa/cm200910/cmselect/cmlonreg/349/349.pdf

14 Mayhew, L. and Harper. G. (2008). Estimating and profiling the population of Hackney. Mayhew Associates Ltd. Online at: www.hackney.gov.uk/Assets/Documents/estimating-andprofiling-the-population-of-hackney.pdf

15 Verrall, R. (2009). Using administrative data to estimate the population and measure deprivation: Full research report. ESRC End of Award report. RES-163-27-0019, Swindon, ESRC.

16 Rees, P., Norman, P. and Brown, D. (2004). A framework for progressively improving small area population estimates. Journal of the Royal Statistical Society, Series A. 167, Pt 1, 5-36.

17 Smith, S. K. and Mandell, M. (1984). A comparison of population estimation methods: Housing unit versus component II, ratio correlation, and administrative records. Journal of the American Statistical Association, Vol. 79, No. 386, 282-289.

18 Smith, Stanley K. (1986). A review and evaluation of the housing unit method of population estimation. Journal of the American Statistical Association, Vol. 81, No. 394, 287-296.

19 Murdock, S. H. and Nazrul Hoque, M. D. (1995). The effect of undercount on the accuracy of small-area population estimates: Implications for the use of administrative data for improving population enumeration. Population Research and Policy Review, 14, 251-271.

20 Hunsinger, E. (2010). Research on regression-based county population estimates for Colorado. Online at: www.demog.berkeley.edu/ eddieh/RatioCorrelationEstimates/RegressionEstimates.pdf

21 Plyer, A., Bonaguro, J. and Hodges, K. (2009). Using administrative data to estimate population displacement and resettlement following a catastrophic U.S. disaster. Population Environment (2010) 21, 150-175.

22 Boden, P. and Rees, P. (2009). Using administrative data to improve the estimation of immigration to local areas in England. Journal of the Royal Statistical Society Series $A$ (2010), 173, Part 4, pp. 707-731.

23 Smith, P. W. F., Raymer, J. and Giulietti, C. (2009). Combining available migration data in England to study economic activity flows over time. Journal of the Royal Statistical Society Series A (2010), 173, Part 4, pp. 733-753.

24 Raymer, J., Abel, G. and Smith, P. W. F. (2007) Combining census and registration data to estimate detailed elderly migration flows in England and Wales. J. R. Statist. Soc. A, 170, 891-908. 
25 Raymer, J., Smith, P. W. F. and Giulietti, C. (2009) Combining census and registration data to analyse ethnic migration patterns in England from 1991 to 2007. Population Space Place, doi 10.1002/psp.565.

26 Statistics New Zealand. Information about the Population Estimates. Online at: www2.stats.govt.nz/domino/external/omni/omni.nsf/outputs/population+estimates

27 Bycroft, C. (2006). Challenges in estimating populations. New Zealand Population Review, 32(2), 21-47.

28 Statistics Canada. (2010). Annual demographic estimates: census metropolitan areas, economic regions and census divisions, age and sex, 2001 to 2006. Online at www.statcan.gc.ca/pub/91-214-x/2006000/4121628-eng.htm

29 Statistics Canada. (2010). Quality assurance programme of the 2011 Canadian Census of Population. UNECE Expert Group Meeting Population and Housing Censuses.

30 Sailer, P., Weber, M. and Yau, E. (1993). How well can IRS count the population? Internal Revenue Service. Proceedings, Section on Survey Research Methods, American Statistical Association.

31 Everaers, P. C. J. and Van Der Laan, P. (2003). The Dutch system of social statistics: Microintegration of different sources. Expert Group Meeting on Setting the Scope of Social Statistics, United Nations Statistics Division.

32 Central Statistical Office, Poland. (2010). Using administrative sources in the National Census 2011: experiences after pilot census. UNECE Expert Group Meeting Population and Housing Censuses.

33 National Institute of Statistics, France. (2010). Census quality. UNECE Expert Group Meeting Population and Housing Censuses. 


\section{Annex A}

\section{Potential benefits and difficulties associated with producing a register-based Census, or population estimate}

Many of the papers summarised in this article have discussed the potential benefits and difficulties in moving towards a register-based census, which could then be used to produce interim population estimates. While not directly relevant to this article, they have been collated below as they may be of more general interest.

Potential benefits for register-based census and population estimates:

- More timely data

- Reduced respondent burden

- Considerable cost and manpower savings

- A consistent time series of population estimates being derived from the same source

- Eliminating inaccuracies from the inter-censal period

- Improving the amount and level of detail that could be produced each year

- Improved estimation of both internal and international migration

- Could serve as a platform to include other sources of admin data, leading to an improved level of detailed information at the local level (see section on micro-integration in the main paper)

- More comprehensive and internally consistent demographic, housing and socio-economic data

- Can be produced for a wide range of low-level geographies, with high precision

- An improved source of data for users

- A reduced number of surveys

Potential difficulties associated with register-based census and population estimates:

- Some loss of information in Census year

- Accounting for the differences observed in population counts between de facto and de jure population definitions. (That is, from counting everyone in the country regardless of usual residence, to counting everyone who is usually resident in the country, including migrants who have been resident for a year or more)

- Improving cooperation between government departments for data sharing

- Applying stringent confidentiality and security measures

- Aligning definitions and classifications across Government (for which the Statistical Office in Singapore took the lead when they encountered this problem)

- Identifying and correcting erroneous records and coverage

- Ensuring that all sources used for the census relate specifically to the agreed census date

- For register-based censuses which are boosted by a sample survey there is the risk of bias if the sample is not directly representative of the total population

- Confidentiality issues; the US Census Bureau are addressing this by looking at laws, rules and procedures 
- Issues around privacy; countries need to make a dedicated effort to build public and institutional trust in order to have a register-based census

- Initial cost; the US Census Bureau is researching lessons learned from other countries that have moved from traditional censuses to administrative records, for example, Finland. While there would need to be high costs initially to set up this approach to population estimates, it is generally thought that a long-term benefit would be reduced costs

- The need for a set of unified identification codes to match and merge records from different administrative systems; US Census Bureau are researching the methods that Finland has developed 